\title{
Facial Expression Recognition Based Scoring System for Restaurants using Deep Learning ()
}

\author{
Nagini K, Lakshmidhar Reddy D, Mokshagna M, Lohith Kumar B, Chetana Tukkoji
}

\begin{abstract}
Now a days, the technology has been increased. Hence this is a tool for the advancement of the earlier things. So we are making use of the technology for restaurant scoring system. The fame of unmanned restaurants is the hot topic in society. Because of the absence of the staff, there is no direct contact with the customers to take feedback of the restaurant. This paper represents the automated rating system for the restaurants by detecting the facial emotions with the help of Convolutional Neural Network [CNN] .It consists of web server and a pretrained CNN and detection of facial expression.

Keywords: CNN, Facial Expression Recognition, web server, Restaurant Scoring.
\end{abstract}

\section{INTRODUCTION}

Facial emotion is most powerful and natural signs for human beings to express their emotional States and feelings,Numerous studies have been conducted on automatic facial emotion analysis,since it is most important in social robotics,medical treatment,driver fatigue surveillance and many other human computer interaction systems.Through the proper vision of machine learning,variety of facial expression recognition systems have been invented to encode the expression information from facial representations as early as the twentieth century,Ekman and Friesen[3] defined six basic expressions based on Cross Culture Study [4] which indicated that human perceive certain basic feelings or emotions regardless of culture.These prototypical facial expressions are anger, disgust, fear, happiness, sadness, and surprise. Contempt was subsequently added as one of the basic emotions [2]. Recently, advanced research on neuroscience and psychology argued that the model of six basic emotions are culture-specific and not universal [3].

In this project we are going to give the rating for the food and the environment of the restaurant by just inputting the image. The designed platform receives the image and detects the facial expression of the image and displays the emotion whether we are happy, satisfied or disappointed with the food or with the environment. For this we are using a platform for input and output.

\subsection{OBJECTIVES}

1. Avoids errors in the data input process and show the correct direction to the management for getting correct information from the computerized system.

Revised Manuscript Received on March 25, 2020.

*Correspondence Author

Nagini K, Assistant Professor of GITAM Deemed To Be University, Computer Science and Engineering.

Lakshmidhar Reddy D, Assistant Professor of GITAM Deemed To Be University, Computer Science and Engineering.

Mokshagna M, Assistant Professor of GITAM Deemed To Be University, Computer Science and Engineering.

Lohith Kumar B, Assistant Professor of GITAM Deemed To Be University, Computer Science and Engineering.

Chetana Tukkoji, Assistant Professor of GITAM Deemed To Be University, Computer Science and Engineering.

(C) The Authors. Published by Blue Eyes Intelligence Engineering and Sciences Publication (BEIESP). This is an open access article under the CC BY-NC-ND license (http://creativecommons.org/licenses/by-nc-nd/4.0/)
2. User-friendly screens for the data entry to handle large volume of data.

3. Unmanned task can be done by just detecting the Facial emotion of the person.

\subsection{REQUIREMENT_ANALYSIS}

The project involved analyzing the design of few applications so as to make the application more users friendly. To do so, it was really important to keep the navigations from one screen to the other well ordered and at the same time reducing the amount of typing the user needs to do. In order to make the application more accessible, the browser version had to be chosen so that it is compatible with most of the Browsers.

\subsubsection{Software Specification}

For developing the application the following are the Software Requirements:

\section{i. Python}

Python is an interactive,object oriented and simple syntax programming language.This programming language express concepts in lesser number of lines. Python interpreters are available for many operating systems. Python supports for multiprogramming paradigms which includes object oriented,functional and imperative library to code the concepts.

\section{ii. Django}

DJango is the the web server that uses python platform.It is a open source web framework which encourages clean design.It takes care of web development,so we can focus on writing source code.It is maintained by DJango foundation.Django main goal is to simplify the creation of complex database driven websites.Django highlights reusability and pluggability of components.Python is even used to set files and data models.

Operating Systems supported

1. Windows 7

2. Windows XP

3. Windows 8

Technologies and Languages used to Develop

1. Python

Debugger and Emulator

Any Browser (Particularly Chrome)

\subsubsection{Hardware Specification}
1. System : Pentium IV $2.4 \mathrm{GHz}$.
2. Hard Disk : $40 \mathrm{~GB}$.
3. Floppy Drive : $1.44 \mathrm{Mb}$.
4. Monitor : 14' Colour Monitor.
5. Mouse : Optical Mouse.
6. Ram : $512 \mathrm{Mb}$ 


\section{LITERATURE SURVEY}

\subsection{EXISTING SYSYEM}

As there is no staff available in unmanned restaurants, it is difficult for the restaurant management to estimate how the concept and the food is experienced by the customers. Existing rating systems, such as Google and TripAdvisor, only partially solve this problem, as they only cover a part of the customer's opinions. These rating systems are only used by a subset of the customers who rate the restaurant on independent rating platforms on their own initiative. This applies mainly to customers who experience their visit as very positive or negative [8].

\subsection{PROPOSED SYSTEM}

In order to solve the above problem, all customers must be motivated to give a rating. This paper introduces an approach for a restaurant rating system that asks every customer for a rating after their visit to increase the number of ratings as much as possible. This system can be used unmanned restaurants; the scoring system is based on facial expression detection using pre trained convolutional neural network (CNN) models. It allows the customer to rate the food by taking or capturing a picture of his face that reflects the corresponding feelings. Compared to text-based rating system, there is much less information and no individual experience reports collected. However, this simple fast and playful rating system should give a wider range of opinions about the experiences of the customers with the restaurant concept.

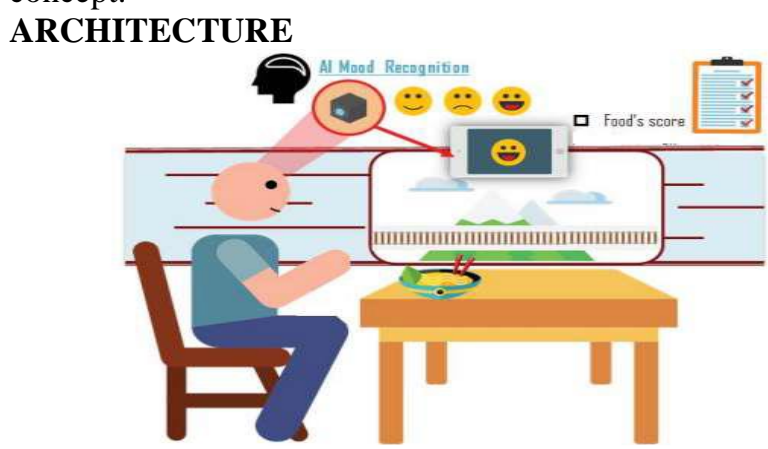

Fig.1 Detection of facial Expression

An interface was created between the user and the admin. Through the interface the user can upload the image. From the image of the person our designed platform detects the expression and displays the output in the form of rating and emotion of the person.

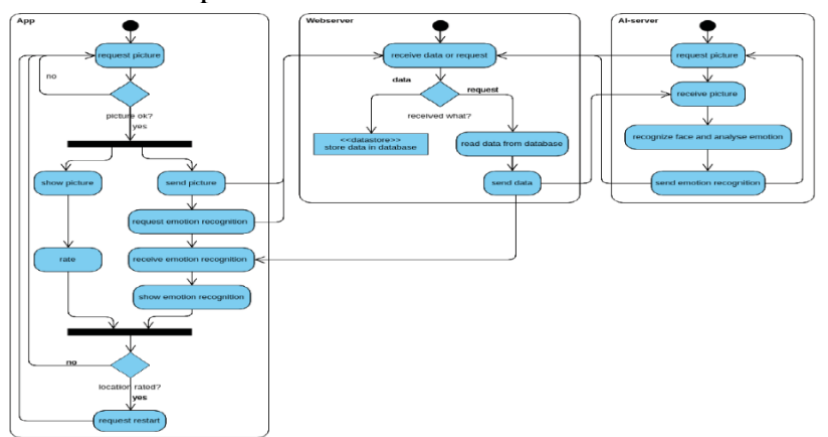

Fig.2 Data Flow Diagram of Restaurant rating system.

The Fig 2 shows how we input the image of the person and then how the server detects the image person and gives output as the emotion of the person and the rating.

\section{RESULTS AND DISCUSSION}

\subsection{FACE DETECTION}

Face expression detection is the most important phase for image classification since,the principal component analysis of nose,mouth,eyes are needed for the classification.Facial detection algorithms are classified based on knowledge feature template models.This paper uses object detection algorithm for facial expression recognition.In this algorithm,Haar Cascade Classifier is used based on is this input image is detected with the help of Haar features.

\subsection{Facial Expression Recognition Sclassification:}

The last step of facial expression recognition is to classify the given input image into any one of the basic emotions such as happy,sad,angry etc.Instead of feature extraction step,the feature classification step are independent.FER is performed in end to end way.Especially,the last layer is is added to the networks and to monitor the errors.[7]

We have design this as a web application using python DJANGO web server. This application can run on user browser where he can upload his photo with rating, uploaded photo will be sent to webserver where machine learning algorithm will be used to extract expression from photo and then saved result to MYSQL database. Another user called 'admin' can login to application and see all users visited to restaurant and can view all customer feedback with facial expression and photo. By seeing this result admin can understand whether customers are happy with their services and foods or not[9][10].

To run this project install MYSQL and then create database by copying content from 'DB.txt' file and paste in MYSQL. Install python and then install DJANGO web server and deploy code on DJANGO. After deployment start server and run the code from browser.

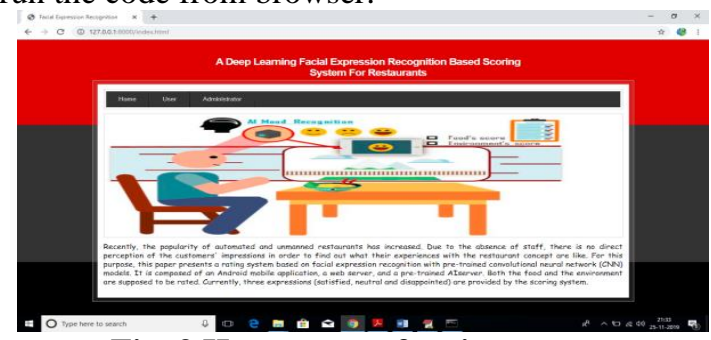

Fig. 3 Home page of rating system

In Fig 3, click on 'User' link to get below screen where user can upload photo and give ratings

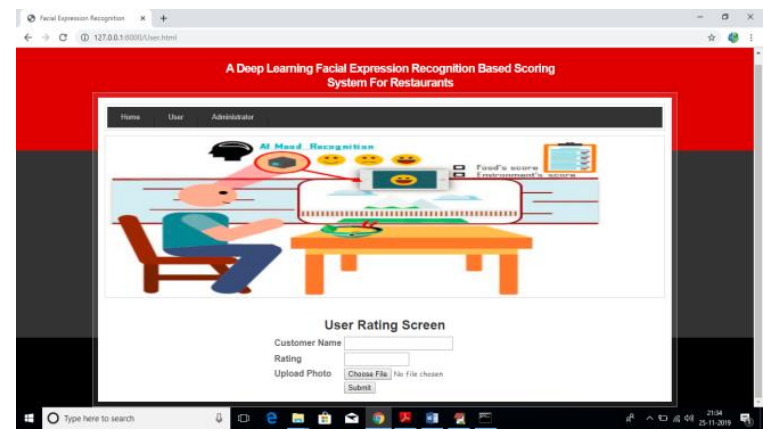

Fig 4 user rating system screen page

From the Fig 4,User will fill above from and upload photo 


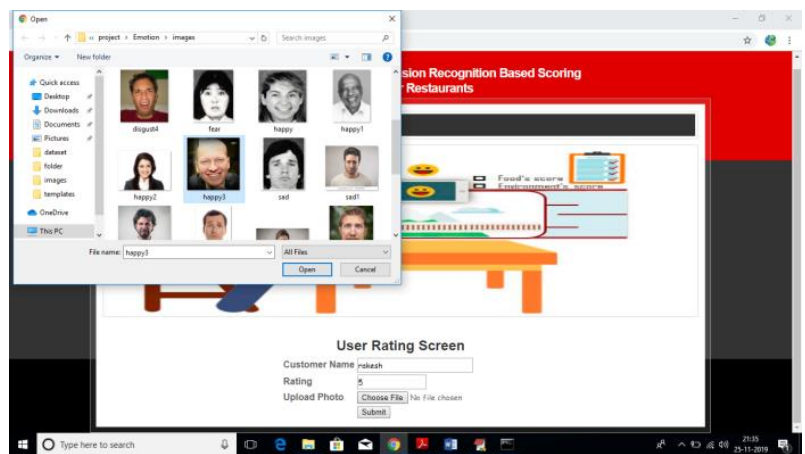

Fig 5 Uploading the image of the person

In Fig 5, user filled form and uploading one happy image and then click on 'Open' button and then click 'Submit' button to send data to webserver. After processing above data will get below results.

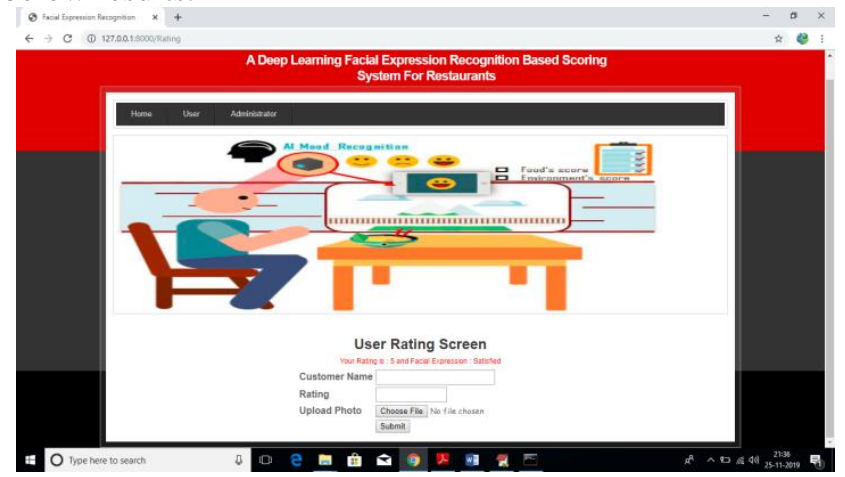

Fig 6 displaying the rating

In Fig 6, we can see output message as given rating and from photo extracted facial expression is satisfied. Now go to 'Administrator' link and login as admin by giving username as 'admin' and password as 'admin'. See below screen.

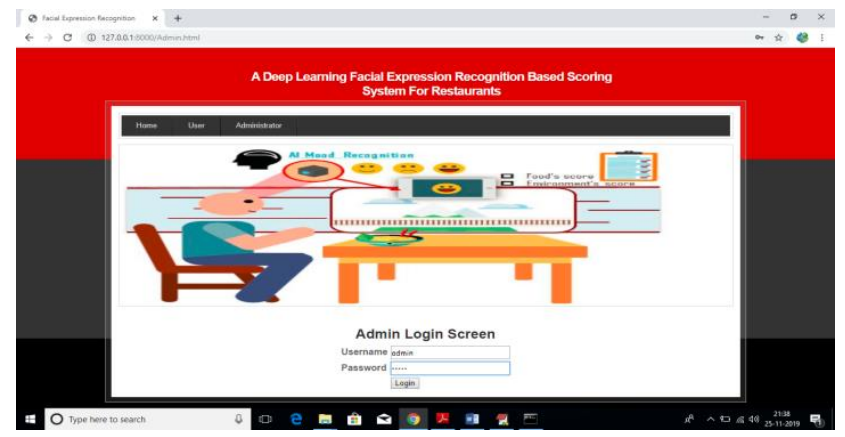

Fig 7 Admin login Page

From the Fig 7, Admin can login into his portal to view the ratings given by the different users.

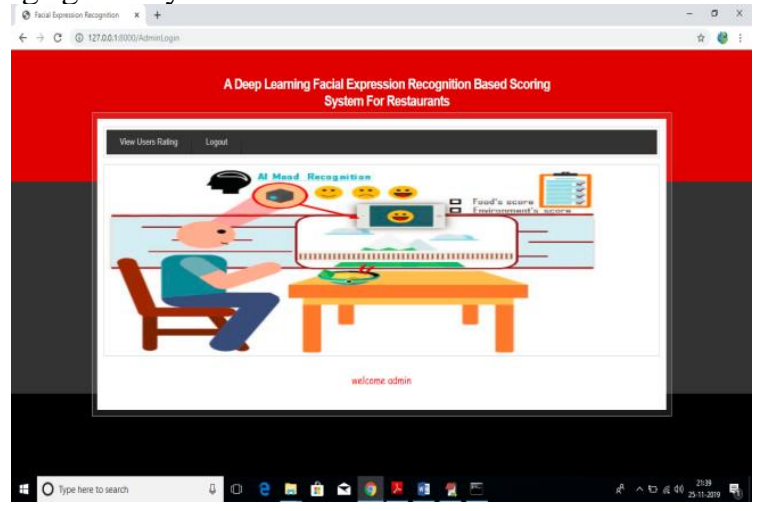

Fig 8 welcome page of admin
In Fig 8, admin can click on 'View Users Rating' link to get all customers feedback. See the Fig 9

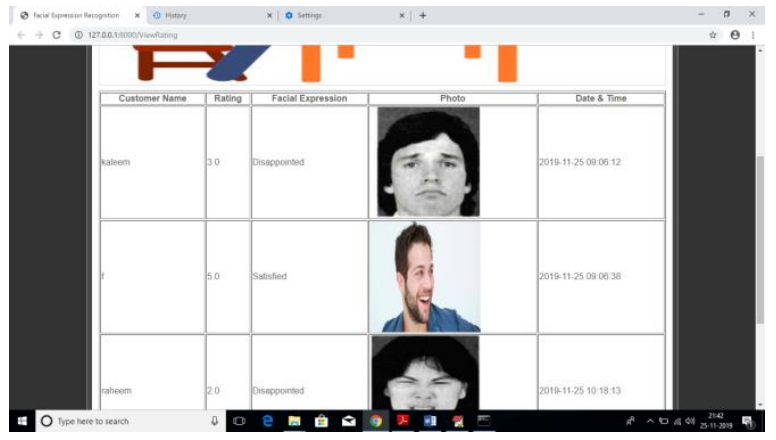

Fig 9 ratings given by various users

From the Fig 9, admin can see photos and their facial expressions

\section{CONCLUSION}

In this paper, rating system for the restaurant was introduced by recognizing the facial expression by inputing the image scanned and principal component analysis was done and compared which Haar features. Now the image is classified and detected whether it is happy or sad or anger. In this paper basically for restaurant scoring we are recognising facial expression and classified into happy satisfied and disappointed.

\section{REFERENCES}

1. Hussain Saeed,Ali Shouman,Malis Elfar,Mostafa Shabka,Shikharesh Majumdar,and Chung Horng-Lung, "Near-field communication sensors and cloud based smart restaurant management system," in Proceedingsof the 2016 IEEattern Recognition(CVPRE $3^{\text {rd }}$ World Forum on Internet of Things(WT-IOT),pp.686-691,2016

2. Florian Schroff,Dmitry Kalenichenko, and James Philbin,'FaceNet:a unified embedding for face recognition and clustering" $n$ Proceedings of the 2015 IEEE Conference on Computer Vision and Pattern Recognition(CVPR),pp.815-823,2015

3. Andrew G. Howard,Menglong zhu,Bo Chen,Dmitry Kalenichenko, Weijun Wang,Tobias sWeyand,Macro Andreeto,and Hartwig Adam,"MobileNets:efficient convolutional neural networks for mobile vision applications,"

4. Janne Tommala,Pedram Ghazi,Bishwo Adhikara,and Hekki Huttunen,"Real time system for facial analysis," in Proceedings of the $7^{\text {th }}$ European Workshop on Visual Information Processing.

5. https://becominghuman.ai/smart-face-control-how-facial-recognitionchanges-different-industries

6. Muscles Of The Face And Their Functions Facial Expression Recognition (Face recognition Techniques) Part $1 \mathrm{M}$ Anatomy "Muscles of the Face and Their Functions facial Expression Recognition (Face Recognition Techniques) Part 1."

7. FERA 2015-Second Facial Expression Recognition and Analysis challenge - IEEE Conference publication

8. Dr. Chetana Tukkoji et.al "ITM-CLD: Intelligent traffic management to handling cloudlets of the large data", published in CSOC 2018-Cybernetics and Algorithms in Intelligent Systems, book series AISC- volume 765, 17 May 2018.

9. Dr. Chetana Tukkoji et.al "Distribution of Data Handling in Cloud Asset", International journal of engineering and advanced technology, volume-9, issue-3 Feb 2020

10. Facial Expression Recognition Using Local Binary Pattern (LBP ) A Saputra T A B Wirayuda A. Saputra and T. A. B. Wirayuda, "Facial Expression Recognition Using Local binary Pattern ( LBP )," 2015

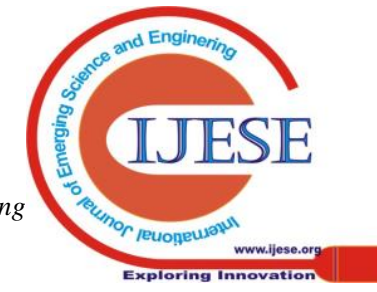


Facial Expression Recognition Based Scoring System for Restaurants using Deep Learning

\section{AUTHORS PROFILE}

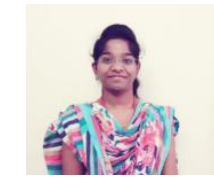

Ms. Nagini K, currently pursuing final year B.Tech degree in Computer Science and Engineering from GITAM deemed to be University, Bengaluru. Her area of interests are Data Science, DBMS, Cloud Computing, and Python.

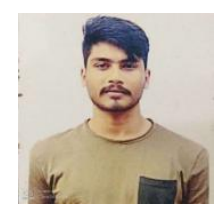

Mr. Lakshmidhar Reddy D, currently pursuing final year B.Tech degree in Computer Science and Engineering from GITAM deemed to be University, Bengaluru. His area of interests are Data Science, DBMS, and Computer Network.

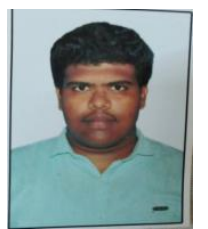

Mr. Mokshagna M, currently pursuing final year B.Tech degree in Computer Science and Engineering from GITAM deemed to be University, Bengaluru. His area of interests are C Programming, Data Science.

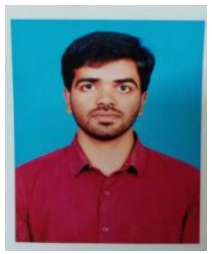

Mr. Lohith Kumar B, currently pursuing final year B.Tech degree in Computer Science and Engineering from GITAM deemed to be University, Bengaluru. His area of interests are C Programming, Data Science, Data Structures, Machine Learning, and Python.

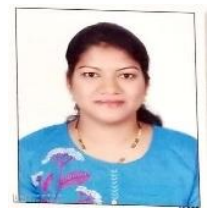

Dr. Chetana Tukkoji, obtained her Bachelor's degree in Computer Science and Engineering from Visvesvaraya technological University, KLECET, Belgavi in 2009. Then she obtained her Master's degree in Computer Science and Engineering from BVBCET, Hubli in 2012 and completed $\mathrm{PhD}$ in Cloud Computing-Big Data Domain from Visvesvaraya Technological University in 2019.Currently she is working as an Assistant Professor of Department of Computer Science in GITAM deemed to be University, Bengaluru. Her areas of interest include Cloud Computing, Computer Network, Data Mining, Robotic Process Automation and Artificial Intelligence. She is having 8 years of teaching and one year of industry experience. She has also published 20 journals including National/International and two books online on C programming for beginners and Computer Organization and Architecture. 